\title{
Varying Experiences of Cyber Victimization among Middle and High School Students
}

\author{
Kathleen I. Díaz ${ }^{1}$ (D) Paula J. Fite ${ }^{1} \cdot$ Madelaine R. Abel $^{1} \cdot$ Rachel L. Doyle $^{1}$
}

Accepted: 7 April 2021 / Published online: 16 April 2021

(C) This is a U.S. government work and not under copyright protection in the U.S.; foreign copyright protection may apply 2021

\begin{abstract}
Background Although cyber victimization (CV) occurs in both middle school (MS) and high school (HS) — and these experiences appear to differ between boys and girls— to our knowledge, no studies have directly examined these differences across specific acts of CV. Further, limited research has examined school environment factors, such as school safety and attachment, as they relate to $\mathrm{CV}$.

Objectives The current study compared CV experiences reported by boys and girls in both MS and HS as well as examined CV's association with perceived school safety and school attachment.

Method Participants were 286 MS and 304 HS students (52\% boys) from a small, rural Midwestern community in the United States. Self-reported measures were collected.

Results HS girls reported experiencing more CV than MS girls on 5 of the $6 \mathrm{CV}$ acts examined. Additionally, HS girls reported experiencing more CV on 3 of the acts compared to MS boys. In general, HS boys and HS girls report similar rates of CV, with the exception of HS girls experiencing higher levels of "people saying mean and nasty things about them." Regression analyses indicated that youth who report higher CV feel less connected to school, but their CV experiences do not appear to be related their perceived school safety when also considering traditional forms of victimization.

Conclusions CV experiences are higher for HS girls for the majority of different types of $\mathrm{CV}$ acts compared to MS youth but similar to HS boys, and experiencing these acts is associated with less school connectedness.
\end{abstract}

Keywords Cyber victimization $\cdot$ Perceived school safety $\cdot$ School attachment $\cdot$ Peer victimization

Kathleen I. Díaz

kathleen.diaz@ku.edu

Paula J. Fite

pfite@ku.edu

1 Clinical Child Psychology Program, 2012 Dole Human Development Center, University

of Kansas, 1000 Sunnyside Avenue, Lawrence, KS 66045, USA 


\section{Introduction}

Cyber victimization (CV), a form of peer victimization (PV), is a hurtful and potentially harmful act that occurs via the Internet or electronic media (Kowalski \& Limber, 2007; Mason, 2008). This prevalent concern among middle and high school (MS, HS) youth has exclusively been examined using mean scores or cut-off scores (e.g., Brown et al., 2017; Díaz \& Fite, 2019), which limits a thorough examination of the varying CV acts that may be occurring as well as whether specific CV acts may differ for boys and girls in MS compared to HS (Baldry et al., 2017; Gardella et al., 2017). Evaluation of the specific acts of CV experienced for boys and girls in MS compared to HS can inform targeted schoolbased interventions, especially since there is preliminary research suggesting that youth who experience victimization are also likely to report school-related problems (Jackson \& Cohen, 2012; Mishna et al., 2010). Thus, the current study examines patterns of CV among predominantly White, gender binary MS boys and girls compared to HS boys and girls, and further evaluates how CV experiences are associated with perceived school safety and school attachment.

\section{Cyber Victimization}

Cyber victimization (CV) has gained much attention over the last decade due to its growing prevalence rate (DePaolis \& Wlliford, 2015; Modecki, et al., 2014; Wang et al., 2009). Youth are increasingly using social media platforms, texting, and other electronic media as primary ways to communicate with each other. Additionally, individuals and schools have increased technology use due to COVID-19, especially as schools have transitioned to online platforms or hybrid formats during the pandemic. As a result of these increases in online communication, youth are at greater risk of increasing rates of bullying and victimization via these cyber and electronic outlets.

Additionally, CV acts can include being called names, being excluded by others, or being threatened via texts or online messages (Del Ray et al., 2015). These acts, in nature, are different from one another and youth may be experiencing different $\mathrm{CV}$ acts at various rates (e.g., experiencing just one or multiple at the same time). However, CV studies have exclusively examined CV mean scores (Dempsey et al., 2009) or have used arbitrary cut-off scores indicating "clinical significance" (Yang et al., 2013). By using these types of grouping methods, the varying degrees of $\mathrm{CV}$ and types of $\mathrm{CV}$ acts that youth may be experiencing are not being captured. For example, a total score of " 2 " on a measure of $\mathrm{CV}$ might represent that the individual received hurtful texts or was the victim of rumors spread via the Internet. A single frequency or severity score does not capture the variability in actual CV acts experienced. Thus, it is unclear whether there are certain CV acts that are more or less common among youth.

When evaluating these differences, it is important to consider various factors, such as race and ethnicity, gender, gender identity, and school level that may put youth at risk for experiencing CV. Given the nature of this sample, this study focused specifically on the association among CV, school level (i.e., MS versus HS), and gender. The current literature has found that CV occurs as early as elementary school (DePaolis \& Williford, 2015) and remains a concern among MS and HS youth (Chan \& La Greca, 2016; Diaz \& Fite, 2019; Schneider et al., 2012). Further, there is some evidence suggesting that MS youth 
experience CV at a higher rate than HS youth (Mishna et al., 2012). As such, CV acts likely differ from MS to HS, but more research is needed to evaluate the rates by which MS compared to HS students experience the distinct acts of CV.

Further, in studies that use binary gender categories, findings on the association between gender and CV have been mixed (Gardella et al., 2017). Some studies have found higher CV rates among boys (Wang et al., 2010) while other studies have found higher rates for girls (Dempsey et al., 2009), and still some studies suggest that there are no gender differences at all (e.g., Brown et al., 2014). One of the few studies to consider gender and grade level differences found that girls reported higher CV during early to mid-adolescence, whereas boys reported higher CV during late adolescence (Barlett \& Coyne, 2014). Thus, gender may be an important factor to consider when evaluating the type and frequency of $\mathrm{CV}$ acts among MS and HS youth. However, this is the first study to examine rates of specific $\mathrm{CV}$ acts among MS and HS boys and girls.

Of note, extant literature has also documented an association between $\mathrm{CV}$ and traditional forms of peer victimization (PV; i.e., physical PV and relational PV). For example, Raskauskas and Stoltz (2007) found that victims of traditional PV were also likely to be victimized via electronic methods (i.e., CV). Similar to other forms of victimization, victims of CV may know their perpetrators. However, unlike the other forms of PV, CV's perpetrators can remain anonymous and spread rumors and false information to as little as one person and up to many youths simultaneously in one incident. Accordingly, studies have found that there are many individuals who are victims of CV but not victims of the traditional forms of PV (Espelage \& Swearer, 2004; Mitchell et al., 2007). For this reason, $\mathrm{CV}$ is a related, yet distinct form of PV. While CV has been found to share many correlates with traditional forms of PV (i.e., physical and relational PV; Chan \& La Greca, 2016; Dempsey et al., 2009), it has also been found to have negative influences on one's functioning, above and beyond youth's experience of traditional PV (Smith, 2012; Wigderson \& Lynch, 2013). Accordingly, the role of traditional forms of PV should be controlled in order to understand the risk and consequences unique to $\mathrm{CV}$.

\section{Correlates of Cyber Victimization}

The social information processing theory (Crick \& Dodge, 1994) has been used to understand the negative influence of PV on youth's social and emotional functioning. According to this framework, individuals encode social stimuli and interpret others' actions based on prior experiences, beliefs, and context. Appraisals of situations, such as cognitive biases and negative attributions, can influence decision-making processes and lead to subsequent behavioral outcomes in response to the perceived intentions of the other person. For example, youth may make hostile attributions about peers (e.g., that peer pushed me on purpose), which may then cause the victim to retaliate. The social information processing theory has recently been applied to CV (Runions et al., 2013). In their model, these authors argue that the anonymous nature of online interactions, lack of accountability, and pervasiveness of Internet use can make it easier to misinterpret others' intentions. For this reason, CV may actually increase the likelihood of hostile attributions errors, leading, in turn, to more negative interactions between peers.

Although CV can occur outside of school, negative interactions between the perpetrator and victim online may also influence in-person interactions at school. There are two possible pathways that may help to explain why CV may influence the school environment. 
First, CV has been associated with high levels of peer rejection and low social acceptability (Wright \& Li, 2013), suggesting that social disconnection may be an important correlate of CV. Second, some studies have found that CV is linked to symptoms of anxiety, depression, and low self-esteem (Dempsey et al., 2009; Kowalski et al., 2014; Landoll et al., 2015; Sinclair et al., 2012; Wang et al., 2011). These factors have been shown to influence one's attitudes about school (Wright, 2015). Due to the important role schooling plays in youth's development, it is vital to understand how experiences of different $\mathrm{CV}$ acts may be associated with one's perception of school safety (i.e., the degree to which youth perceive that going to or being at school is safe; Astor et al., 2009) and school attachment (i.e., the degree to which youth feel connected to their school) as these factors may serve as a protective factor. Thus, the current study extends these models by examining the links between $\mathrm{CV}$ and perceived school safety and school attachment.

To date, studies have found that youth are more likely to feel unsafe in places in which traditional bullying and victimization occurs (Hazler et al., 1992). Youth who report higher levels of traditional victimization also report lower perceptions of safety in school (Slee \& Rigby, 1993). In fact, experiencing PV is consistently associated with lower reports of perceived school safety and school attachment. Specifically, high levels of physical and relational PV were associated with poorer school engagement among younger adolescents (Hoglund, 2007) and was negatively associated with perceived school safety among older adolescents (Astor et al., 2009; Fite et al., 2019; Gower et al., 2015). Additionally, the PV literature has found that a positive perception of the school climate, such as feeling safe in this environment, can buffer the negative consequences associated with PV (Wormington et al., 2016).

Of note, individuals who are targets of traditional PV are often also targets of CV (Juvoven \& Gross, 2008; Ybarra et al., 2007). Given that CV and traditional forms of victimization tend to co-occur and have similar correlates, it seems likely that youth who experience $\mathrm{CV}$ will also report lower perception of school safety. However, due to the lack of research studies on $\mathrm{CV}$, less information is known about the negative correlates of $\mathrm{CV}$, especially perceptions about school safety and school attachment. In fact, while there is increasing evidence that there is some overlap between $\mathrm{CV}$ and the school environment, very limited literature has found that $\mathrm{CV}$ is associated with the perception of school as an unsafe environment (Varjas et al., 2009). One study found that higher levels of CV were associated with lower perceived social support from teachers and peers, peer rejection, and negative school experiences, including perceived discrimination and lower school satisfaction among middle and high schoolers (Evans et al., 2014). Lack of perceived teacher responsiveness to victimization, in particular, may cause youth to perceive school as an unsafe place. Overall, factors such as low school connectedness critically influence school avoidance behaviors (Berkowitz \& Benbenishty, 2012; Hutzell \& Payne, 2012), which, in turn, may lead to disengagement from school and perceptions that school is unsafe or unsupportive. However, this hypothesis is primarily based in theory as very limited literature has explicitly examined this link between CV and the perception of school safety.

Additionally, CV is linked to poor academic performance, school absenteeism, and behavioral concerns at school (Beran \& Li, 2005; Hinduja \& Patchin, 2008; SolomontosKountouri et al., 2017; Wright, 2015). Ybarra et al. (2007) found that youth who experienced CV had more detentions, suspensions, and episodes of truancy than those who did not experience CV. The work of Hinduja and Patchin (2008) found similar results. Specifically, CV among adolescents was positively associated with school problems (e.g., tardiness, truancy). In addition, $\mathrm{CV}$ has been linked to feelings of loneliness, lower rates of social acceptability by peers, and symptoms of depression and anxiety (Dempsey et al., 
2009; Jackson \& Cohen, 2012; Kowalski et al., 2014; Landoll et al., 2015), all of which can influence school performance. These deficits in school functioning-including detentions, truancy, and academic performance-may influence youth's perception of school as an engaging, positive place (e.g., Wang \& Holcombe, 2010). That is, youth who experience CV are at greater risk of negative outcomes relating to school and as such may hold a negative perception about their school as a result. In fact, if the environment is perceived to be hostile, youth may disengage from school, further harming their attachment to, and engagement with, school (Wang \& Holcombe, 2010). However, to our knowledge, no study has directly examined the association between $\mathrm{CV}$ and school attachment.

\section{Current Study}

In order to address the current gaps in the CV literature, this study examined which acts of $\mathrm{CV}$ are experienced among MS boys and MS girls compared to HS boys and HS girls. As noted in previous literature, CV was expected to be prevalent in both MS and HS (Chan \& La Greca, 2016; Diaz \& Fite, 2019); however, given that higher rates of CV were found in MS versus HS (Mishna et al., 2012), it was expected that MS students would report higher $\mathrm{CV}$ rates. Given that most research has examined mean scores for $\mathrm{CV}$, it was not clear what specific acts of CV would be reported at higher rates. Since gender and MS versus HS findings have been mixed, this study posited that gender differences may emerge; however, no specific predictions of the direction of these associations were hypothesized (Barlett \& Coyne, 2014; Mishna et al., 2012). Further, CV was expected to be associated with lower levels of school attachment and perceived school safety, similar to previous PV literature (Slee \& Rigby, 1993) as CV and traditional forms of PV share common correlates (Chan \& La Greca, 2016; Dempsey et al., 2009). As such, physical PVand relational PV were also considered when evaluating the association between $\mathrm{CV}$ and school variables in order to evaluate unique associations.

\section{Methods}

\section{Participants}

As part of a larger institutional review board (IRB) approved study, 286 middle and 304 high school students (52\% boys; $M$ age $=14.03, S D=2.06)$ in a small Midwest school district completed electronic surveys. Gender was examined as a binary construct (boys versus girls), and as a result we were not able to look at group differences for those who identify as gender non-binary. Information on individual participants' sexual orientation, socioeconomic status, language spoken at home, religion, disability status, culture, race, and ethnicity were not available to the research team. However, the race and ethnicity reported for the school was White and non-Hispanic (86\%), Hispanic (5-6\%), Black (1\%), Asian (1\%), American Indian (1-2\%), and two or more races (5\%), which is similar to the city where data was collected (82.9\% White and non-Hispanic; U.S. Census Bureau, 2010). Further, the median household income for the city in which data was collected is roughly $\$ 87,600$ (U.S. Census Bureau, 2010), and neaarly 34\% of youth are eligible for free or reduced lunch in this school district. 


\section{Measures}

\section{Cyber Victimization}

$\mathrm{CV}$ was assessed using selective items from the European Cyberbullying Intervention Project Questionnaire Items (Del Rey et al., 2015) to be consistent with the measure of the traditional forms of PV utilized in the current study. Youth responded to six-items using a 5-point Likert scale $(1=$ Never, $2=$ Once or Twice, $3=A$ Few Times, $4=$ About Once $a$ Week, $5=A$ Few Times a Week). The questionnaire was reworded to assess if CV was experienced since the beginning of the academic year. A sample item is "someone said nasty things to me or called me names using texts or online messages" (all items are reported in Table 1). Item level responses in addition to mean scores were evaluated in the current study, with higher scores representing higher rates of CV experienced. This self-reported scale has been previously found to be a reliable and valid measure of exposure to CV (e.g., Del Rey, et al., 2015) and demonstrated good internal consistency in the current study $(\alpha=0.91)$.

\section{Physical and Relational Forms of Peer Victimization (Traditional Forms of PV)}

Physical and relational forms of victimization were assessed using a downward extension of the Victimization of Self Scale from the Peer Experience Questionnaire (PEQ; Vernberg et al., 1999). This nine-item self-report measure is on a 5-point Likert scale $(1=$ Never, $2=$ Once or Twice, $3=A$ Few Times, $4=$ About Once a Week, 5=A Few Times a Week), with four items addressing physical victimization (e.g., "a kid hit, kicked, or pushed me in a mean way") and five items addressing relational victimization (e.g., "a kid ignored me on purpose to hurt my feelings") since the beginning of the school year. Mean scores were computed and used for analyses, with higher scores indicating more victimization being experienced. The PEQ has been previously found to be a reliable and valid measure of exposure to both physical and relational victimization (e.g., Vernberg et al., 1999) and showed good internal consistency in the current study for physical victimization and relational victimization ( $\alpha=0.81$ and 0.87 , respectively).

\section{Perceived School Safety}

Perceived school safety was assessed using a 5-item measure (Henrich et al., 2004) adapted from the Yale Child Study Center's Social and Health Assessment survey (Schwab-Stone et al., 1995, 1999; Weissberg et al., 1991). This self-report measure is on a 4-point Likert scale $(1=$ Definitely Not True, $2=A$ Little True, 3=Mostly True, $4=$ Definitely True) and asked children to respond to how safe they feel at various school locations (e.g., "I feel safe on the school bus or while walking to school;" "I feel safe in the restrooms at my school"), with higher mean scores indicating greater perceived school safety. Previous research using this scale in youth populations has demonstrated acceptable to good internal consistency (Henrich, et al., 2004). Internal consistency for the measure in the current study was good $(\alpha=0.89)$. 


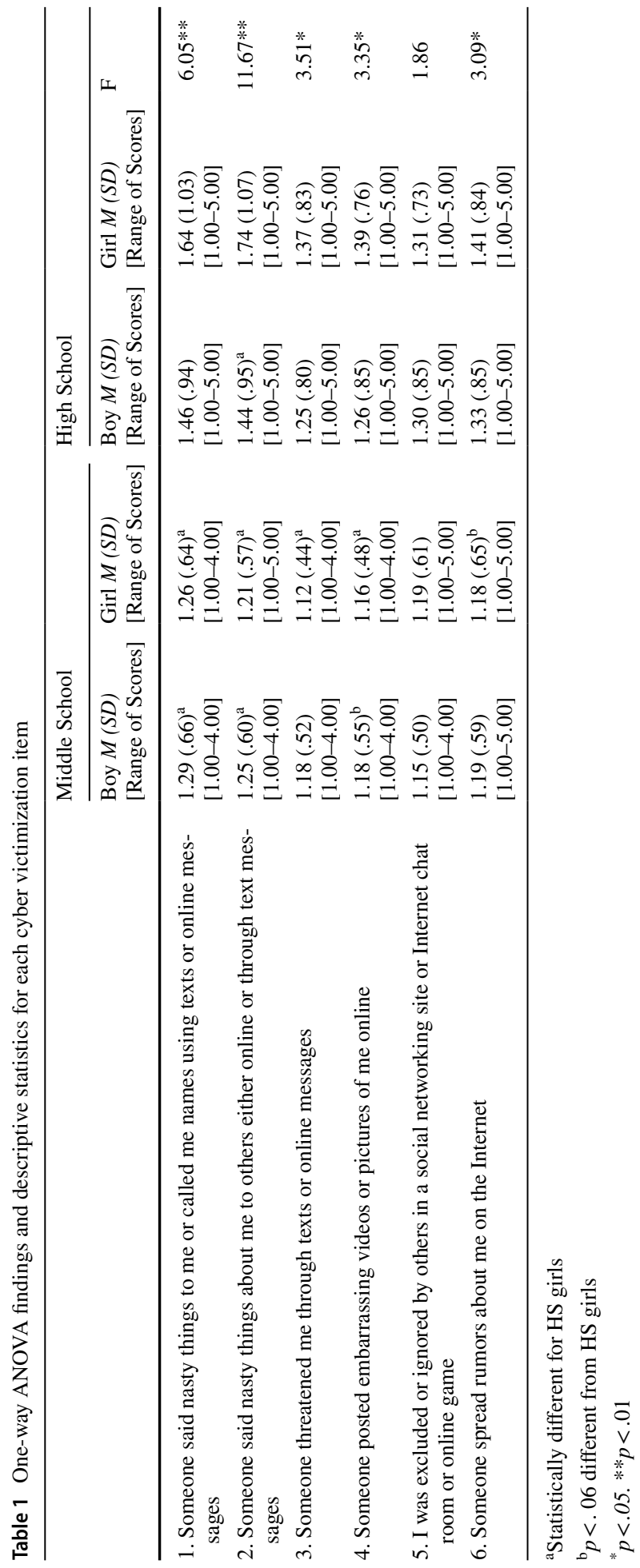




\section{School Attachment}

School attachment was assessed using three items ("I feel close to people at this school;" "I am happy to be at this school;" and "I feel like I am part of this school") from the National Longitudinal Study of Adolescent Health (Libby, 2004; Moody \& Bearman, 1998). This self-report measure is on a 5-point Likert scale (1=Strongly Disagree, 2=Disagree, $3=$ Neutral, $4=$ Agree, $5=$ Strongly Agree) . Higher mean scores indicated higher levels of attachment to school. Previous research using this scale in child populations has demonstrated high reliability and good internal consistency (McNeely et al., 2002). The internal consistency of this scale in the current sample was good $(\alpha=0.83)$.

\section{Procedures}

The researchers' IRB, as well as both MS and HS administrators, approved the current study prior to data collection. Consent was obtained from a legal guardian/parent of each student participant prior to participation in the study. Consent rates were good $(82.06 \%$ for MS and $80.5 \%$ for HS) as were student assent and completion rates (75.7\% for MS and $67.3 \%$ for HS). Students who had consent and gave assent completed a 30-min survey hosted through the confidential online survey platform (Qualtrics, 2017), while trained study personnel read the survey aloud. Data from the MS was collected in the students' classrooms, while data from the HS was collected in large group rooms in which students were spread out (i.e., cafeteria, band room, gymnasium, and library) during the typical school day. Students self-reported their age, grade, and gender while completing their battery of measures. Data collection occurred during the fall semester of the 2017-2018 academic year. No compensation was provided.

\section{Data Analytic Plan}

IBM SPSS Statistics 25 (IBM Corp., 2017) was used to conduct study analyses. First, six separate ANOVAs were conducted to evaluate if there were mean level differences for each $\mathrm{CV}$ item among boys in MS, boys in HS, girls in MS, and girls in HS. Given our interest at the individual item level rather than the overall difference, and to aid in interpretation of post hoc analyses, ANOVAs at the individual item level were conducted in lieu of a MANOVA (Bray et al., 1985). Tukey post hoc tests were used to determine differences among the four groups. Multiple regression analyses were then conducted in to evaluate unique associations between CV and school attachment and perceived school safety, while also considering the variance associated with traditional forms of PV (i.e., physical PV and relational PV). Finally, gender and MS versus HS differences in associations between CV and school attachment and perceived school safety were evaluated by including interaction terms $(\mathrm{CV} \times$ Gender, $\mathrm{CV} \times \mathrm{MS} / \mathrm{HS})$ in the models. Each interaction term was added to the model one at a time. Significant interactions were probed using simple slope analyses at 1 SD above and below the mean, with the models conditioned to represent boys versus girls and MS versus HS, according to standard procedures (Holmbeck, 2002). Listwise deletion was used to account for missing data since less than $5 \%$ of data relevant to the current study was missing. All study variables were standardized prior to conducting regression analyses to aid in the interpretation of interaction effects. 
Effect sizes (r-values, Cohen's d, and $f^{2}$ ) reported describe the magnitude of the associations. Correlation (r-values) effect sizes are characterized by small (0.10), medium (0.30), or large if greater than or equal to 0.50 (Cohen, 1988). ANOVA effect sizes (d values) of 0.2 are considered a small effect, 0.5 a medium effect, and 0.8 a large effect (Cohen, 1988). For significant regression effects, $f^{2}$ effect sizes are characterized as small (0.02), medium (0.15), or large (0.35 or greater; Aiken \& West, 1991; Cohen, 1988).

Post hoc power analyses using G*Power 3.1 (Faul et al., 2009) were conducted to determine the ability to detect effects with the current sample size. The post hoc power analyses conducted examined both ANOVA and linear multiple regressions using two-tailed tests with $\alpha=0.05$. ANOVA models included four different groups and regression models included five to six predictor variables. ANOVA post hoc power analyses indicated that the current sample size was powered $(>0.80)$ to detect medium to large effects. Linear multiple regressions post hoc power analyses also indicated that the current sample was adequately powered $(>0.80)$ to detect medium to large effect sizes.

\section{Results}

\section{Descriptive Statistics}

From the total sample, $39.3 \%$ reported experiencing CV, $40.8 \%$ reported experiencing physical PV, and 54.6\% reported experiencing relational PV. Among the youth in MS, $31.1 \%$ of youth reported experiencing $\mathrm{CV}, 46 \%$ of youth reported experiencing physical $\mathrm{PV}$, and $53 \%$ of youth reported experiencing relational PV. Among the youth in HS, $47 \%$ of youth reported experiencing $\mathrm{CV}, 36 \%$ of youth reported experiencing physical PV, and $56.1 \%$ of youth reported experiencing relational PV. Additionally, $71.2 \%$ of MS youth reported being well attached to the school (mean scores higher than 4 ) whereas $47 \%$ of high school youth reported being well attached to the school. In contrast, middle and high school youth reported similar levels of mostly feeling safe at school (mean scores higher than $3 ; 83.1 \%$ and $81.5 \%$, respectively). Mean scores for the individual CV acts are found in Table 1. The mean scores for the individual acts physical PV and relational PV are found in the Table 2.

\section{Item Level Analyses}

ANOVA analyses found statistically significant mean level group differences on 5 of the 6 $\mathrm{CV}$ acts. Those acts included: "Someone said nasty things about me to others either online or through text messages" $(\mathrm{F}(3,567)=11.67, p<0.01 ; \mathrm{d}=0.07$ between MS boys and MS girls, $\mathrm{d}=0.30$ between HS boys and HS girls, $\mathrm{d}=0.24$ between MS boys and HS boys, and $\mathrm{d}=0.62$ between MS girls and HS girls), "Someone said nasty things to me or called me names using texts or online messages" $(\mathrm{F}(3,570)=6.05, p<0.01 ; \mathrm{d}=0.05$ between MS boys and MS girls, $\mathrm{d}=0.18$ between HS boys and HS girls, $\mathrm{d}=0.21$ between MS boys and HS boys, and $\mathrm{d}=0.44$ between MS girls and HS girls), "Someone posted embarrassing videos or pictures of me online" $(\mathrm{F}(3,569)=3.35, p=0.02 ; \mathrm{d}=0.04$ between MS boys and girls, $\mathrm{d}=0.16$ between HS boys and girls, $\mathrm{d}=0.11$ between MS boys and HS boys, and $\mathrm{d}=0.36$ between MS girls and HS girls), "Someone threatened me through texts or online messages" $(\mathrm{F}(3,570)=3.51, p=0.02 ; \mathrm{d}=0.13$ between MS boys and MS girls, $\mathrm{d}=0.15$ 


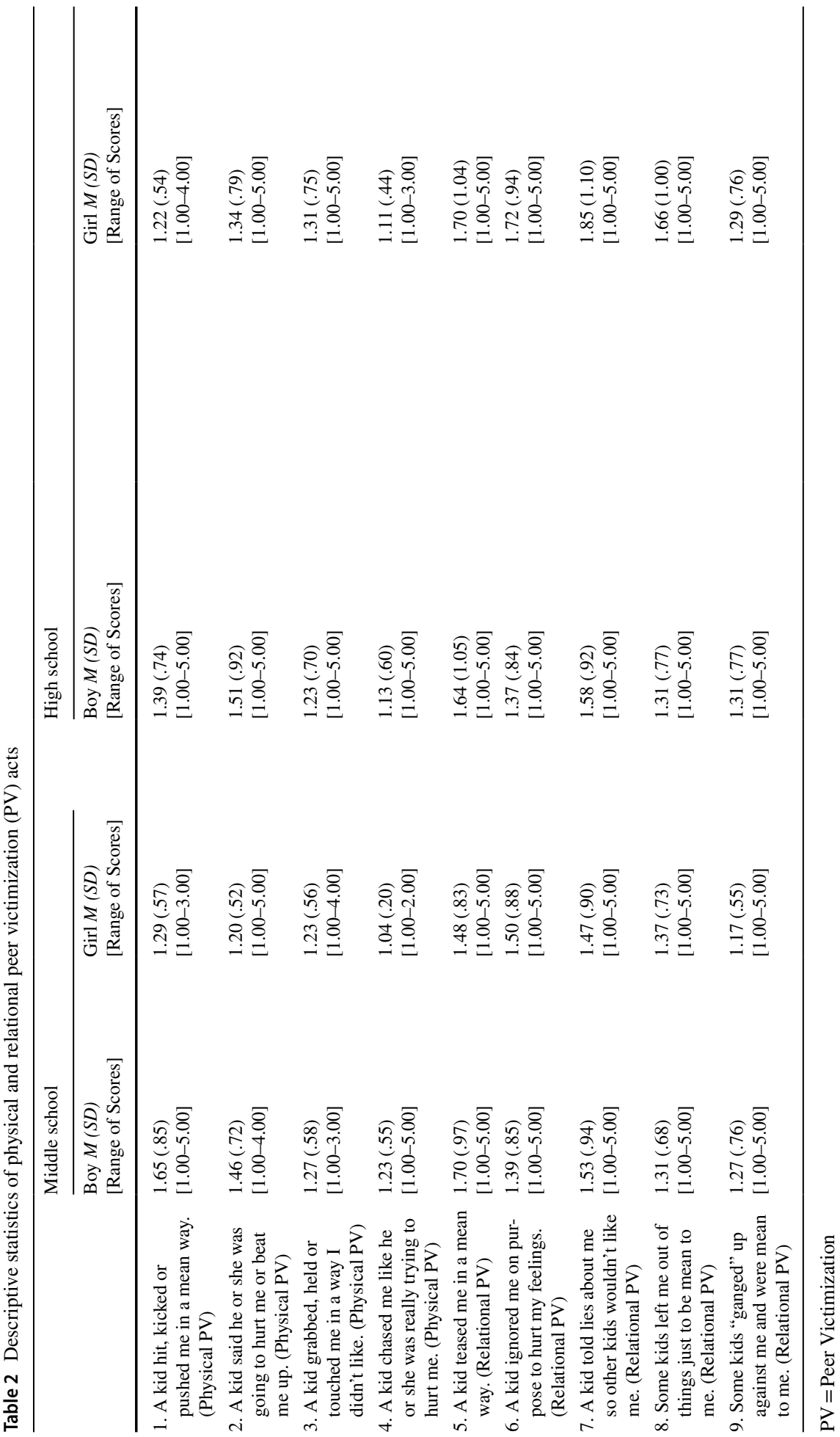


between HS boys and HS girls, $\mathrm{d}=0.10$ between MS boys and HS boys, and $\mathrm{d}=0.38$ between MS girls and HS girls), and "Someone spread rumors about me on the internet" $(\mathrm{F}(3,569)=3.09, p=0.03 ; \mathrm{d}=0.02$ between MS boys and MS girls, $\mathrm{d}=0.09$ between HS boys and HS girls, $\mathrm{d}=0.19$ between MS boys and HS boys, and $\mathrm{d}=0.31$ between MS girls and HS girls).

Post hoc analyses (see Table 1) indicated that HS girls reported higher levels of "Someone said nasty things about me to others either online or through text messages" than any other group of students. HS girls also reported higher levels of "Someone said nasty things to me or called me names using texts or online messages" than MS boys and MS girls. HS girls reported higher levels of "Someone posted embarrassing videos or pictures of me online" than MS girls, and there was a marginally statistically significant trend for MS boys to report lower levels of this item than HS girls. Finally, HS girls reported higher levels than MS girls on the item "Someone threatened me through texts or online messages," and a marginally statistically significant trend on the item "Someone spread rumors about me on the internet."

\section{Associations Between Forms of Victimization and School Related Outcomes}

Correlation analyses (see Table 3) indicated that all 3 forms of victimization (physical PV, relational $\mathrm{PV}$, and $\mathrm{CV}$ ) were significantly negatively associated with both perceived school safety $(r(579)=-0.38, p<0.01 ; r(579)=-0.35, p<0.01 ; r(583)=-0.30, p<0.01$, respectively) and school attachment $(r(579)=-0.23, p<0.01 ; r(579)=-0.32, p<0.01$; $r(583)=-0.30, p<0.01$, respectively). Additionally, boys reported more physical peer victimization $(r(569)=-0.14, p=0.001)$ and lower perceived school safety $(r(569)=-0.10$, $p=0.02)$ than girls. HS students reported higher levels of CV $(r(583)=0.17, p<0.01)$ and lower levels of school attachment $(r(583)=-0.13, p=0.002)$ than MS students. CV, relational PV, and physical PV were strongly positively associated with one another (sharing up to $49 \%$ of their variance), indicating related but distinct victimization types.

Multiple regression analyses were then conducted to evaluate unique associations (see Table 4). The first model conducted examined the association between school attachment $\left(f^{2}=0.16\right)$ while the second model conducted examined the association between perceived

Table 3 Correlations coefficients, means, and standard deviations for all study variables

\begin{tabular}{llllllll}
\hline & 1 & 2 & 3 & 4 & 5 & 6 & 7 \\
\hline 1. Gender & & & & & & & \\
2. MS/HS & -.05 & & & & & & \\
3. Cyber Victimization & .03 & $.17^{* *}$ & & & & & \\
4. Physical Peer Victimization & $-.14^{* *}$ & -.03 & $.63^{* *}$ & & & & \\
5. Relational Peer Victimization & .06 & .08 & $.70^{* *}$ & $.66^{* *}$ & & & \\
6. Perceived School Safety & $.10^{*}$ & .01 & $-.30^{* *}$ & $-.38^{* *}$ & $-.35^{* *}$ & & \\
7. School Attachment & .05 & $-.13^{* *}$ & $-.30^{* *}$ & $-.23^{* *}$ & $-.32^{* *}$ & $.40^{* *}$ & \\
$M$ & & & 1.30 & 1.28 & 1.47 & 3.63 & 4.06 \\
SD & & & .63 & .52 & .71 & .52 & .90 \\
\hline
\end{tabular}

Gender ( 1 =Boys, 2 = Girls). MS/HS (1=Middle School, $2=$ High School $)$

${ }^{*} p<.05 . * * p<.01$ 


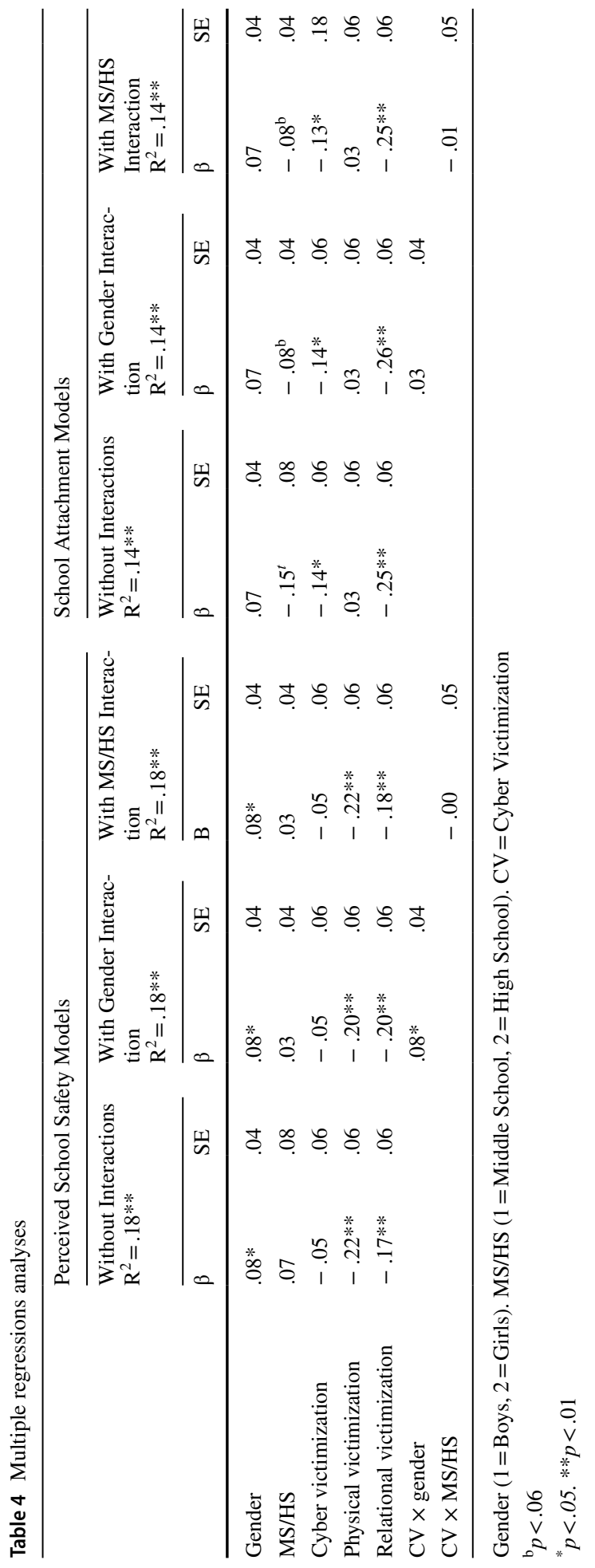


school safety $\left(f^{2}=0.21\right)$. Findings indicated that $\mathrm{CV}$ was uniquely associated with school attachment $(\beta=-0.14, p=0.02)$ but not perceived school safety $(\beta=-0.05, p=0.38)$, with high levels of CV associated with lower levels of school attachment. Relational PV was uniquely associated with both school attachment $(\beta=-0.25, p<0.001)$ and perceived school safety $(\beta=-0.17, p<0.001)$, with high levels of relational PV associated with lower levels of school attachment and lower levels of perceived school safety. Physical PV was associated with perceived school safety $(\beta=-0.22, p<0.001)$ but not school attachment $(\beta=0.03, p=0.63)$, with high levels of physical PV associated with lower levels of perceived school safety. Girls reported higher levels of perceived school safety than boys. Finally, there was a marginally statistically significant trend for MS students to report higher levels of school attachment than HS students $(\beta=-0.15, p=0.06)$. In sum, CV and relational PV were significantly associated with perceived school attachment, whereas only relational PV and physical PV were significantly associated with perceived school safety.

\section{Gender and School Level Differences in Associations Between CV and School Related Outcomes}

Gender and MS versus HS differences in associations between CV and school related outcomes were evaluated by examining interaction effects. No differences between MS and HS students were evident in associations between CV and school attachment $(\beta=-0.01$, $p=0.81)$ or perceived school safety $(\beta=-0.002, p=0.97)$. Additionally, gender did not moderate the association between $\mathrm{CV}$ and school attachment $(\beta=0.03, p=0.47)$.

In contrast, a statistically significant $\mathrm{CV} \times$ Gender interaction was found for perceived school safety $\left(\beta=0.08, p=0.03, f^{2}=0.22\right)$. However, the test of simple slopes indicated that the association between $\mathrm{CV}$ and perceived school safety was not statistically significant for boys $(\beta=-0.13, p>0.06)$ or girls $(\beta=0.04, p=0.59)$. These findings suggest that although the associations are statistically different for boys and girls, CV is not statistically significantly associated with perceived school safety for boys or girls.

\section{Discussion}

The current study extends the CV literature in multiple ways. First, this study examined the varying acts of $\mathrm{CV}$ among MS boys and girls compared to HS boys and girls. To our knowledge, no studies could be found that evaluate these differences. Secondly, this study examined whether youths' CV experiences were associated with school-related outcomes, such as perceived school safety and school attachment. This is incredibly important given the increase usage of technology, especially as a result of the COVID-19 pandemic.

Consistent with previous literature (Wigderson \& Lynch, 2013), this study found that $\mathrm{CV}$ occurs in both MS and HS. Interestingly, however, HS girls experienced the majority (5 of 6 ) of $\mathrm{CV}$ acts at a higher rate than MS girls. Further, HS girls experienced acts of nasty things being said about them via texts and other online messaging as well as posting embarrassing images more often than MS boys; yet MS boys and HS girls experienced acts of exclusion, threats, and rumors online and via the Internet at a similar rate. It appears that the differences are the use of texts and online messages, which are more prevalent for HS girls than MS boys. In contrast, HS girls and HS boys reported similar rates among most $\mathrm{CV}$ acts, with the exception of HS girls reporting that 
individuals said nasty things about them to others more often than HS boys. Further, MS boys and MS girls as well as HS boys are experiencing similar rates of CV acts. Although the mean scores of the various CV experiences were low, HS girls experienced the highest rates of most acts of CV. Yet, the rates at which boys and girls experience various acts of $\mathrm{CV}$ are similar in both MS and HS. Thus, although HS girls are experiencing higher rates of various CV acts than MS girls, they are not experiencing starkly higher rates than their HS male counterparts. It may be that HS youth use text and online messages more frequently as a means of communication compared to MS youth, resulting in these differences, or that more HS youth have their own phone and laptop, whereas perhaps MS youth use a school-provided or family computer. Additionally, it may be that caregivers more closely monitor the text messages of their MS youth than their HS youth, making it more possible for CV to occur via text messages in HS. Overall results are in contrast to Mishna and colleagues (2012) study that found that MS youth report higher rates of CV than HS youth. It is possible that the differences in results may be due to sample characteristics, such as the area in which these youth live (i.e., a small rural area) and being a predominantly White sample. Alternatively, differences may be related to $\mathrm{CV}$ assessed globally as opposed to rates of specific types of $\mathrm{CV}$. Accordingly, more research examining rates of $\mathrm{CV}$ acts across MS and $\mathrm{HS}$ is needed before firm conclusions can be drawn regarding school level effects.

Previous research regarding gender differences in rates of $\mathrm{CV}$ have been mixed (e.g., Gardella et al., 2017). Current findings are consistent with prior research that found no gender differences in CV for MS youth (Brown et al., 2014), and further suggest that rates of specific acts are similar for males and females in MS and HS. Nonetheless, additional research is needed to further evaluate gender differences given the mixed findings in the literature. Further, it should be noted that in this study gender was examined as a binary variable (boy/girl) and did not include non-binary gender identification, which may be an important consideration for future studies especially given the limited literature on this topic to date. However, this study provides preliminary evidence that gender and school level may be two important factors that influence rates and experiences of CV.

In addition, CV was uniquely associated with school attachment but not perceived school safety. Specifically, higher levels of CV were related to lower school attachment when also considering rates of traditional PV, and these effects were similar for MS and HS and boys and girls. This finding is consistent with previous literature that suggests that CV is associated with lower school satisfaction (Evans et al., 2014). Consistent with the social information processing theory (Crick \& Dodge, 1994) and the work of Runions and colleagues (2013), it is possible that youth who experience CV may form negative associations (i.e., hostile attribution errors) towards their school because they may not feel supported and cared for by school personal or peers (Eliot et al., 2010). This may be especially true if their peers are the preparators. In the current study, a similar pattern emerged with relational PV. Youth who reported higher relational PV also endorsed feeling less attached to school.

This study also found that only the traditional forms of PV, not CV, were uniquely associated with perceived school safety, such that youth who reported higher physical PV or relational PV also reported lower perceived school safety. This aligns with previous literature that states youth who experience traditional forms of PV are also likely to view this environment as unsafe (Hazler et al., 1992). It may be that because CV often occurs outside of the school context that experiencing $\mathrm{CV}$ does not make one feel particularly unsafe in the school environment. 


\section{Limitations, Implications, and Future Directions}

This study has limitations worth noting. First, this study was limited in the demographic information collected (i.e., no data regarding SES or individual youth race and ethnicity). Further, the study sample was relatively homogenous, consisting of gender binary and mostly White $(>85 \%)$ youth, rendering generalizability to more diverse populations unclear. Previous studies that have examined CV among more diverse samples have found ethnic and racial minority school-aged to high-school aged youth reported higher rates of both traditional PV and CV (Barlett \& Wright, 2018). Similarly, non-gender binary youth are also at greater risk of experiencing peer victimization and bullying (Norris \& Orchowski, 2020). Thus, more literature is needed regarding CV experiences and how these experiences may also vary among more diverse individuals groups. Further, data were cross-sectional, preventing us from establishing causal or predictive effects. Previous $\mathrm{PV}$ and CV research found that rates of both PV and CV tend to decrease over the course of the school year (Pellegrini \& Bartini, 2000; Pellegrini \& Long, 2002). It is possible that the current study's results may have varied if a longitudinal approach was also examined. Finally, given potential power concerns (and limited ability to detect small effects), a 3-way interaction was not conducted to examine if a certain CV act, MS/HS, and gender in one model influenced the association among perceived school safety and school attachment. Future studies should consider these limitations in order to better understand CV acts that are endorsed and CV's association with perceived school safety and attachment.

Despite these limitations, findings suggest that in general HS boys and HS girls experience similar levels of $\mathrm{CV}$ - with the exception of people saying mean and nasty things about them, with HS girls reporting higher rates than HS boys. However, HS girls experience more $\mathrm{CV}$ than MS girls on 5 of the $6 \mathrm{CV}$ behaviors examined, suggesting possible age differences and that $\mathrm{CV}$ is occurring more for girls in HS than MS. Additionally, HS girls reported more $\mathrm{CV}$ on 3 of the acts than MS boys, indicating that HS girls experience more $\mathrm{CV}$ than MS boys and MS girls, particularly in acts of texts and online messages. In addition, when considering CV's impact on school, those who report higher CV are feeling less connected to school, but their CV experiences do not appear to influence their perceived school safety when also considering the variance associated with traditional forms of PV.

These results can aid in intervention efforts to reduce the rates and the impact of CV. While older youth are more likely to have more access to electronic means, it is possible that these findings are also due to different monitoring of technology in the home and school. Although CV does not require in-person contact, school interventions may still buffer the negative effects of CV-including not feeling attached to school. For example, adults at school (i.e., teachers, counselors) may help to mitigate the impact of $\mathrm{CV}$ on school connectedness. In addition, policies at school regarding social media use may help to prevent $\mathrm{CV}$ exposure during the school day. Given that $\mathrm{CV}$ experiences in HS are fairly similar among boys and girls, intervention efforts may not need to consider gender-specific adaptations to decrease the hurtful and potentially harmful acts occurring via the Internet or electronic media. That is, both HS boys and HS girls may benefit from similar strategies during this school level. CV rates and types differed between MS girls and HS girls and thus school might consider targeting specific interventions depending on girls' grade level. Taken together, this study highlights the importance of examining individual CV acts as well as emphasizes the importance of continual examination of school-related outcomes that CV may influence. 


\section{Declarations}

Conflict of interest The authors have no conflicts of interest to report.

Human Participants and/or Animals Research involved human subjects, with all procedures approved by the Institutional Review Board (IRB).

Informed Consent Informed parental consent and child assent were obtained for all study participants.

\section{References}

Aiken, L. S., \& West, S. G. (1991). Multiple regression: Testing and interpretinginteractions. . Sage.

Astor, R., Benbenishty, R., \& Estrada, J. (2009). School violence and theoretically atypical schools: The principal's centrality in orchestrating safe schools. American Educational Research Journal, 46(2), 423-461. https://doi.org/10.3102/0002831208329598.

Baldry, A. C., Farrington, D. P., \& Sorrentino, A. (2017). School bullying and cyberbullying among boys and girls: Roles and overlap. Journal of Aggression, Maltreatment \& Trauma, 26(9), 937-951. https:// doi.org/10.1080/10926771.2017.1330793.

Barlett, C., \& Coyne, S. M. (2014). A meta-analysis of sex differences in cyber-bullying behavior: The moderating role of age. Aggressive Behavior, 40(5), 474-488. https://doi.org/10.1002/ab.21555.

Barlett, C. P., \& Wright, M. F. (2018). Longitudinal relations among cyber, physical, and relational bullying and victimization: Comparing majority and minority ethnic youth. Journal of Child \& Adolescent Trauma. https://doi.org/10.1007/s40653-017-0174-8.

Beran, T., \& Li, Q. (2005). Cyber-harassment: A study of a new method for an old behavior. Journal of Educational Computing Research, 32, 265-277. https://doi.org/10.2190/8YQM-B04H-PG4D-BLLH.

Berkowitz, R., \& Benbenishty, R. (2012). Perceptions of teachers' support, safety, and absence from school because of fear among victims, bullies, and bully-victims. American Journal of Orthopsychiatry, 82, 67-74. https://doi.org/10.1111/j.1939-0025.2011.01132.x.

Bray, J. H., Maxwell, S. E., \& Maxwell, S. E. (1985). Multivariate analysis of variance (No. 54). . Sage.

Brown, C. F., Demaray, M. K., \& Secord, S. M. (2014). Cyber victimization in middle school and relations to social emotional outcomes. Computers in Human Behavior, 35, 12-21. https://doi.org/10.1016/j. chb.2014.02.014.

Brown, C. F., Demaray, M. K., Tennant, J. E., \& Jenkins, L. N. (2017). Cyber victimization in high school: Measurement, overlap with face-to-face victimization, and associations with social-emotional outcomes. School Psychology Review, 46(3), 288-303. https://doi.org/10.17105/SPR-2016-0004.V46-3.

Chan, S. F., \& La Greca, A. M. (2016). Cyber victimization and aggression: Are they linked with adolescent smoking and drinking?. In Child \& Youth Care Forum (Vol. 45, No. 1, pp. 47-63). Springer US. https://doi.org/https://doi.org/10.1007/s10566-015-9318-x

Cohen, J. (1988). Statistical power analysis for the social sciences. (2nd ed.). Lawrence Erlbaum Associates, Publishers.

Crick, N. R., \& Dodge, K. A. (1994). A review and reformulation of social information-processing mechanisms in children's social adjustment. Psychological Bulletin, 115, 74-101. https://doi.org/10.1037/ 0033-2909.115.1.74.

Del Rey, R., Casas, J. A., Ortega-Ruiz, R., Schultze-Krumbholz, A., Scheithauer, H., Smith, P., Smith, P., Thompson, F., Barkoukis, V., Tsorbatzoudis, H., Brighi, A., Guarini, A., Pyzalski, J., \& Plichta, P. (2015). Structural validation and cross-cultural robustness of the European cyberbullying intervention project questionnaire. Computers in Human Behavior, 50, 141-147. https://doi.org/10.1016/j.chb. 2015.03.065.

Dempsey, A., Sulkowski, M., Nichols, R., \& Storch, E. (2009). Differences between peer victimization in cyber and physical settings and associated psychological adjustment in early adolescence. Psychology in the Schools, 46, 962-972. https://doi.org/10.1002/pits.20437.

DePaolis, K., \& Williford, A. (2015). The nature and prevalence of cyber victimization among elementary school children. In Child \& Youth Care Forum (Vol. 44, No. 3, pp. 377-393). Springer US. https://doi. org/https://doi.org/10.1007/s10566-014-9292-8

Díaz, K. I., \& Fite, P. J. (2019). Cyber victimization and its association with substance use, anxiety, and depression symptom among middle school youth. Child and Youth Care Forum. https://doi.org/10. 1007/s10566-019-09493-w. 
Eliot, M., Cornell, D., Gregory, A., \& Fan, X. (2010). Supportive school climate and student willingness to seek help for bullying and threats of violence. Journal of School Psychology, 48(6), 533-553. https:// doi.org/10.1016/j.jsp.2010.07.001.

Espelage, D. L., \& Swearer, S. M. (Eds.). (2004). Bullying in American schools: A social-ecological perspective on prevention and intervention. . Routledge. https://doi.org/10.4324/9781410609700.

Evans, C. B. R., Smokowski, P. R., \& Cotter, K. L. (2014). Cumulative bullying victimization: An investigation of the dose-response relationship between victimization and the associated mental health outcomes, social supports, and school experiences of rural adolescents. Children and Youth Services Review, 44, 256-264. https://doi.org/10.1016/j.childyouth.2014.06.021.

Faul, F., Erdfelder, E., Buchner, A., \& Lang, A. G. (2009). Statistical power analyses using G*Power 3.1: Tests for correlation and regression analyses. Behavior Research Methods, 41(4), 1149-1160. https:// doi.org/10.3758/BRM.41.4.1149.

Fite, P. J., Poquiz, J., Díaz, K. I., Williford, A., \& Tampke, E. C. (2019). Links between peer victimization, perceived school safety, and internalizing symptoms in middle childhood. School Psychology Review, 48(4), 309-319. https://doi.org/10.17105/SPR-2018-0092.V48-4.

Gardella, J. H., Fisher, B. W., \& Teurbe-Tolon, A. R. (2017). A systematic review and meta-analysis of cyber-victimization and educational outcomes for adolescents. Review of Educational Research, 87(2), 283-308. https://doi.org/10.3102/0034654316689136.

Gower, A. L., McMorris, B. J., \& Eisenberg, M. E. (2015). School-level contextual predictors of bullying and harassment experiences among adolescents. Social Science and Medicine, 147, 47-53. https://doi. org/10.1016/j.socscimed.2015.10.036.

Hazler, R. J., Hoover, J. H., \& Oliver, R. (1992). What kids say about bullying. The Executive Educator, 14, 20-22.

Henrich, C. C., Schwab-Stone, M., Fanti, K., Jones, S. M., \& Ruchkin, V. (2004). The association of community violence exposure with middle-school achievement: A prospective study. Journal of Applied Developmental Psychology, 25(3), 327-348. https://doi.org/10.1016/j.appdev.2004.04.004.

Hinduja, S., \& Patchin, J. W. (2008). Cyberbullying: An exploratory analysis of factors related to offending and victimization. Deviant Behavior, 29(2), 129-156. https://doi.org/10.1080/01639620701457816.

Holmbeck, G. N. (2002). Post-hoc probing of significant moderational and mediational effects in studies of pediatric populations. Journal of Pediatric Psychology, 27(1), 87-96. https://doi.org/10.1093/jpepsy/ 27.1.87.

Hoglund, W. L. (2007). School functioning in early adolescence: Gender-linked responses to peer victimization. Journal of Educational Psychology, 99(4), 683. https://doi.org/10.1037/0022-0663.99.4.683.

Hutzell, K. L., \& Payne, A. A. (2012). The impact of bullying victimization on school avoidance. Youth Violence and Juvenile Justice, 10, 370-385. https://doi.org/10.1177/1541204012438926.

IBM Corp. Released. (2017). IBM SPSS Statistics for Windows, Version 25.0. Armonk, NY: IBM Corp.

Jackson, C. L., \& Cohen, R. (2012). Childhood victimization: Modeling the relation between classroom victimization, cyber victimization, and psychosocial functioning. Psychology of Popular Media Culture, 1(4), 254-269. https://doi.org/10.1037/a0029482.

Juvonen, J., \& Gross, E. F. (2008). Extending the school grounds? Bullying experiences in cyberspace. Journal of School Health, 78, 496-505. https://doi.org/10.1111/j.1746-1561.2008.00335.x.

Kowalski, R. M., Giumetti, G. W., Schroeder, A. N., \& Lattanner, M. R. (2014). Bullying in the digital age: A critical review and meta-analysis of cyberbullying research among youth. Psychological Bulletin, 140, 1073-1137. https://doi.org/10.1037/a0035618.

Kowalski, R. M., \& Limber, S. P. (2007). Electronic bullying among middle school students. Journal of Adolescent Health, 41, S22-S30. https://doi.org/10.1016/j.jadohealth.2007.08.017.

Landoll, R. R., La Greca, A. M., Lai, B. S., Chan, S. F., \& Herge, W. M. (2015). Cyber victimization by peers: Prospective associations with adolescent social anxiety and depressive symptoms. Journal of Adolescence, 42, 77-86. https://doi.org/10.1016/j.adolescence.2015.04.002.

Libby, H. P. (2004). Measuring student relationships to school: Attachment, bonding, connectedness, and engagement. Journal of School Health, 74(7), 274-283. https://doi.org/10.1111/j.1746-1561.2004. tb08284.x.

Mason, K. L. (2008). Cyberbullying: A preliminary assessment for school personnel. Psychology in the Schools, 45(4), 323-348. https://doi.org/10.1002/pits.20301.

McNeely, C. A., Nonnemaker, J. M., \& Blum, R. W. (2002). Promoting school connectedness: Evidence from the national longitudinal study of adolescent health. Journal of School Health, 72(4), 138-146. https://doi.org/10.1111/j.1746-1561.2002.tb06533.x.

Mitchell, K. J., Ybarra, M., \& Finkelhor, D. (2007). The relative importance of online victimization in understanding depression, delinquency, and substance use. Child Maltreatment, 12(4), 314-324. https://doi.org/10.1177/1077559507305996. 
Mishna, F., Cook, C., Gadalla, T., Daciuk, J., \& Solomon, S. (2010). Cyber bullying behaviors among middle and high school students. American Journal of Orthopsychiatry, 80(3), 362-374. https://doi.org/10. 1111/j.1939-0025.2010.01040.x.

Mishna, F., Khoury-Kassabri, M., Gadalla, T., \& Daciuk, J. (2012). Risk factors for involvement in cyber bullying: Victims, bullies and bully-victims. Children and Youth Services Review, 34(1), 63-70. https://doi.org/10.1016/j.childyouth.2011.08.032.

Modecki, K. L., Minchin, J., Harbaugh, A. G., Guerra, N. G., \& Runions, K. C. (2014). Bullying prevalence across contexts: A meta-analysis measuring cyber and traditional bullying. Journal of Adolescent Health, 55(5), 602-611. https://doi.org/10.1016/j.jadohealth.2014.06.007.

Moody, J., \& Bearman, P. S. (1998). Shaping school climate: School context, adolescent social networks, and attachment to school. Unpublished manuscript.

Norris, A. L., \& Orchowski, L. M. (2020). Peer victimization of sexual minority and transgender youth: A cross-sectional study of high school students. Psychology of Violence, 10(2), 201. https://doi.org/ 10.1037/vio0000260.

Pellegrini, A. D., \& Bartini, M. (2000). A longitudinal study of bullies, victims, and peer affiliation during the transition from primary school to middle school. American Educational Research Journal, 37, 699-725. https://doi.org/10.3102/00028312037003699.

Pellegrini, A. D., \& Long, J. (2002). A longitudinal study of bullying, dominance, and victimization during the transition from primary to secondary school. British Journal of Developmental Psychology, 20, 259-280. https://doi.org/10.1348/026151002166442.

Qualtrics (2017). Provo, UT: Qualtrics

Raskauskas, J., \& Stoltz, A. D. (2007). Involvement in traditional and electronic bullying among adolescents. Developmental Psychology, 43(3), 564. https://doi.org/10.1037/0012-1649.43.3.564.

Runions, K., Shapka, J. D., Dooley, J., \& Modecki, K. (2013). Cyber-aggression and victimization and social information processing: Integrating the medium and the message. Psychology of Violence, 3 , 9-26. https://doi.org/10.1037/a0034503.

Schneider, S. K., O’Donnell, L., Stueve, A., \& Coulter, R. W. (2012). Cyberbullying, school bullying, and psychological distress: A regional census of high school students. American Journal of Public Health, 102(1), 171-177. https://doi.org/10.2105/AJPH.2011.300308.

Schwab-Stone, M., Ayers, T. S., Kasprow, W., Voyce, C., Barone, C., Shriver, T., \& Weissberg, R. P. (1995). No safe haven: A study of violence exposure in an urban community. Journal of the American Academy of Child \& Adolescent Psychiatry, 34(10), 1343-1352. https://doi.org/10.1097/00004 583-199510000-00020.

Schwab-Stone, M., Chen, C., Greenberger, E., Silver, D., Lichtman, J., \& Voyce, C. (1999). No safe haven II: The effects of violence exposure on urban youth. Journal of the American Academy of Child \& Adolescent Psychiatry, 38(4), 359-367. https://doi.org/10.1097/00004583-19990 4000-00007.

Sinclair, K. O., Bauman, S., Poteat, V. P., Koenig, B., \& Russell, S. T. (2012). Cyber and bias-based harassment: Associations with academic, substance use, and mental health problems. Journal of Adolescent Health, 50, 521-523. https://doi.org/10.1016/j.jadohealth.2011.09.009.

Slee, P. T., \& Rigby, K. (1993). Australian school children's self appraisal of interpersonal relations: The bullying experience. Child Psychiatry and Human Development, 23, 273-282. https://doi.org/10. 1007/BF00707680.

Smith, P. K. (2012). Cyberbullying and cyber aggression. In S. R. Jimerson, A. B. Nickerson, M. J. Mayer, \& M. J. Furlong (Eds.), Handbook of school violence and school safety. International Research and Practice, 2. (pp. 93-103). New York: Routledge.

Solomontos-Kountouri, O., Tsagkaridis, K., Gradinger, P., \& Strohmeier, D. (2017). Academic, socioemotional and demographic characteristics of adolescents involved in traditional bullying, cyberbullying, or both: Looking at variables and persons. International Journal of Developmental Science, 11, 19-30. https://doi.org/10.3233/DEV-17219.

U.S. Census Bureau. (2010). Explore Census Data. https://data.census.gov/cedsci/

Varjas, K., Henrich, C. C., \& Meyers, J. (2009). Urban middle school students' perceptions of bullying, cyberbullying, and school safety. Journal of School Violence, 8, 159-176. https://doi.org/10.1080/ 15388220802074165 .

Vernberg, E. M., Jacobs, A. K., \& Hershberger, S. L. (1999). Peer victimization and attitudes about violence during early adolescence. Journal of Clinical Child Psychology, 28, 386-395. https://doi.org/ $10.1207 / \mathrm{S} 15374424 \mathrm{jccp} 280311$.

Wang, M. T., \& Holcombe, R. (2010). Adolescents' perceptions of school environment, engagement, and academic achievement in middle school. American Educational Research Journal, 47, 633662. https://doi.org/10.3102/0002831209361209. 
Wang, J., Iannotti, R. J., \& Nansel, T. R. (2009). School bullying among adolescents in the United States: Physical, verbal, relational, and cyber. Journal of Adolescent Health, 45(4), 368-375. https://doi. org/10.1016/j.jadohealth.2009.03.021.

Wang, J., Iannotti, R. J., Luk, J. W., \& Nansel, T. R. (2010). Co-occurrence of victimization from five subtypes of bullying: Physical, verbal, social exclusion, spreading rumors, and cyber. Journal of Pediatric Psychology, 35(10), 1103-1112. https://doi.org/10.1093/jpepsy/jsq048.

Wang, J., Nansel, T. R., \& Iannotti, R. J. (2011). Cyber and traditional bullying: Differential association with depression. Journal of Adolescent Health, 48, 415-417. https://doi.org/10.1016/j.jadohealth.2010. 07.012 .

Weissberg, R. P., Voyce, C. K., Kasprow, W. J., Arthur, M. W., \& Shriver, T. P. (1991). The social and health assessment. . Authors.

Wigderson, S., \& Lynch, M. (2013). Cyber-and traditional peer victimization: Unique relationships with adolescent well-being. Psychology of Violence, 3(4), 297. https://doi.org/10.1037/a0033657.

Wormington, S. V., Anderson, K. G., Schneider, A., Tomlinson, K. L., \& Brown, S. A. (2016). Peer victimization and adolescent adjustment: Does school belonging matter? Journal of School Violence, 15(1), 1-21. https://doi.org/10.1080/15388220.2014.922472.

Wright, M. F. (2015). Adolescents' cyber aggression perpetration and cyber victimization: The longitudinal associations with school functioning. Social Psychology of Education, 18, 653-666. https://doi.org/10. 1007/s11218-015-9318-6.

Wright, M. F., \& Li, Y. (2013). The association between cyber victimization and subsequent cyber aggression: The moderating effect of peer rejection. Journal of Youth and Adolescence, 42, 662-674. https:// doi.org/10.1007/s10964-012-9903-3.

Yang, S. J., Stewart, R., Kim, J. M., Kim, S. W., Shin, I. S., Dewey, M. E., Maskey, S., \& Yoon, J. S. (2013). Differences in predictors of traditional and cyber-bullying: A 2-year longitudinal study in Korean school children. European Child \& Adolescent Psychiatry, 22(5), 309-318. https://doi.org/10.1007/ s00787-012-0374-6.

Ybarra, M. L., Diener-West, M., \& Leaf, P. J. (2007). Examining the overlap in internet harassment and school bullying: Implications for school intervention. Journal of Adolescent Health, 41(6), S42-S50. https://doi.org/10.1016/j.jadohealth.2007.09.004.

Publisher's Note Springer Nature remains neutral with regard to jurisdictional claims in published maps and institutional affiliations. 